\title{
Pyrexia of unknown origin: a rare presentation of primary ovarian lymphoma
}

\author{
Asha N. Gokhale ${ }^{1}$, Akriti Agarwal ${ }^{1 *}$, Bharat D. Purandare ${ }^{2}$
}

\begin{abstract}
${ }^{1}$ Department of Obstetrics and Gynecology, Deenanath Mangeshkar Hospital and Research Centre, Pune, Maharashtra, India

${ }^{2}$ Department of Infectious Diseases, Deenanath Mangeshkar Hospital and Research Centre, Pune, Maharashtra, India
\end{abstract}

Received: 06 March 2020

Accepted: 30 March 2020

\section{*Correspondence:}

Dr. Akriti Agrwal,

E-mail: agarwalakriti8@gmail.com

Copyright: ( $)$ the author(s), publisher and licensee Medip Academy. This is an open-access article distributed under the terms of the Creative Commons Attribution Non-Commercial License, which permits unrestricted non-commercial use, distribution, and reproduction in any medium, provided the original work is properly cited.

\begin{abstract}
It is very rare to have a lymphomatous involvement of ovary. Malignant lymphoma of ovary is a well-known late manifestation of disseminated nodal disease. Primary ovarian lymphoma with ovarian mass as an initial manifestation is a rare entity and may have varied presentations which can cause confusion to the physician and cause delay in diagnosis. Study presents a case of non-Hodgkin's lymphoma where the initial presentation was fever with weight loss, and was evaluated as pyrexia of unknown origin. When no other cause of fever was identified PET-CT was done showing metabolically active uterine mass with no lymphadenopathy. Exploratory laparotomy was planned followed by hysterectomy with bilateral salpingo ophorectomy with omentectomy. Ovarian malignancy was detected intraoperatively, which was diagnosed as diffuse large B cell lymphoma, NHL double expresser phenotype on histopathology and IHC. Patient was started on chemotherapy and is doing fine.
\end{abstract}

Keywords: Diffuse large B cell lymphoma, Primary ovarian lymphoma, Pyrexia of unknown origin

\section{INTRODUCTION}

It is well-known that cancer, diagnosed or undiagnosed, can cause fever. Indeed, fever from underlying malignancy accounts for up to $25 \%$ of cases of fever of underdetermined origin in some series. ${ }^{1}$ Furthermore, lymphoma is the most common etiology of neoplastic fever of underdetermined origin. The patho-physiology of tumor-induced fever may be due to several mechanisms of which include release of cytokines from tumor cells or infiltrating mononuclear cells. ${ }^{2}$

Cancer fever classically is associated with Hodgkin's lymphoma, but can occur in the setting of non-Hodgkin's lymphoma, leukemia, and solid tumors.

Lymphomas presenting with gynecological symptoms are rare. Ovarian lymphoma has a varied clinical presentation. It may occur de novo or secondary to systemic disease and macroscopically appear as solid ovarian tumors. ${ }^{3}$ NHL involving the ovaries may cause confusion for the clinician since its presentation is varied and may resemble other, much more frequent tumors. The most common presenting signs or symptoms of ovarian lymphomas are abdominal or pelvic pain or mass. ${ }^{4}$ It may also present with ascitis and raised CA $125 .^{5}$ A very rare and atypical presentation with which this case patient presented was fever for last 3 weeks with weight loss, with no other specific symptom making the diagnosis difficult.

\section{CASE REPORT}

A 62-year-old post-menopausal female, P4L4 was referred to the study hospital with a history of fever for past 3 weeks. She had complained of body ache, loss of 
appetite and weight loss of around $5 \mathrm{~kg}$ in a month. There was no history of nausea vomiting, abdominal distention, bowel bladder complains.

She was partially investigated for fever with tests for leptospira, malaria, rickettsia and dengue, all of which were normal. Bone marrow, chest $\mathrm{x}$ ray done which was normal. USG pelvis showed well defined hypo echoic lesion with lobulated margins in pelvis along anterior aspect of uterus $8 \times 5.3 \mathrm{~cm}$, lesion had significant vascularity, mild spleenomegaly. CT abdomen showed uterine fibroid.

With the above history she was admitted and was further investigated as pyrexia of unknown origin.

She was recently diagnosed to be diabetic and was on insulin.

On examination she was pale, had tachycardia. On systemic examination there was no hepatosplenomegaly, per abdomen examination was unremarkable. Per vaginal examination, uterus was bulky, mass of around $8 \mathrm{~cm}$ felt in the left fornix, with a groove separating the mass from uterus. On investigations brucella was positive, was started on doxycycline and piperacillin-tazobactum. Complete blood counts revealed anemia, thrombocytopenia, platelets 64000, mild leucocytosis with monocytosis, and no lymphocytosis. ESR 140, LFT normal. LDH was raised 656, CRP 137, CA-125 13.56.

Was treated for bruclella, antibiotics given, but fever persisted. Hematological consultation was taken, blood transfused, bone marrow slide reviewed from study hospital showed minimally hyper cellular marrow with dimorphic maturation. Thrombocytopenia persisted. After ruling out any infective or non-infective pathology, PETCT was done which showed metabolically active uterine lesion $73 \times 75 \times 72 \mathrm{~mm}$ arising from left lateral uterine wall suggestive of fibroid and diffuse FDG uptake in gall bladder. No retroperitoneal or pelvic lymphadenopathy seen.

In view of the above findings with a diagnosis of uterine malignancy, patient was prepared for exploratory laparotomy.

Intraoperatively degenerating friable mass was seen arising from the left ovary pointing the diagnosis towards solid ovarian tumor. Right ovary and fallopian tube were grossly normal. Peritoneal fluid was sent for cytology, the sample was sent for frozen section. The surgery included total hysterectomy with $\mathrm{B} / \mathrm{L}$ salpingo-ophorectomy and omentectomy. Lymph node dissection not done as neither CT nor PET scan showed lymphatic involvement. The entire operated specimen was submitted for histopathological examination.

The frozen section reported it to be high grade malignancy not of epithelial origin, fluid cytology was positive for malignancy, atypical cells with high N/C ratio, and hyper chromatic nuclei with scanty cytoplasm.

The final histopathological report stated, on microscopy, uterus with atrophic endometrium, left ovary showed features of high-grade malignancy, tumor cells loosely arranged in sheets separated with fibrous septa. Cells were small to intermediate in size with monotonous hyper chromatic nuclei and scant cytoplasm. Right ovary, fallopian tube and omentum appeared unremarkable and free of tumor.

IHC done (Figure 1) and found tumor cells positive for LCA, CD 20, CD 10, Bcl-2, Bcl-6, Mum-1. 50-55\% of the cells show nuclear c-myc positivity. Mib-1 index was $90 \%$. Tumor cells were negative for CK, vimentin, CD-3, and CD-5. Final diagnosis was made as high-grade Bcell Non-Hodgkin's lymphoma - Germinal cell type with double expresser phenotype. CD20 and LCA (a Pan B cell marker) was positive in tumor cells and cytokeratin and CD3 were negative. The tumor was double expresser type, positive for Bcl-2, and myc.

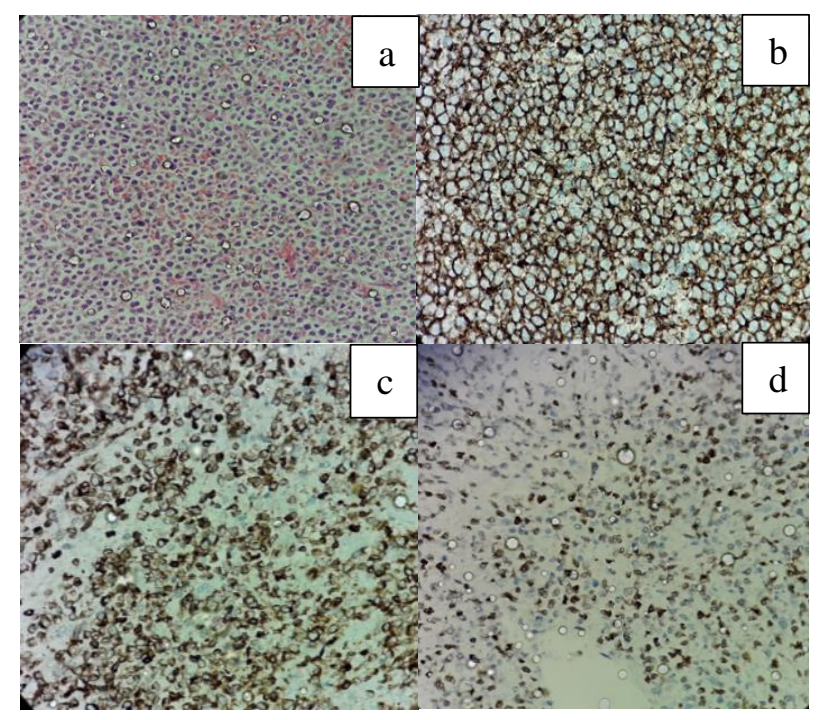

Figure 1: Diffuse infiltration of malignant lymphoid cells (a) $\mathrm{H}$ and $\mathrm{E}$ stain, (b) CD 20 stained, (c) Bcl 2 and (d) C-Myc on IHC.

Postoperatively, patient was started on chemotherapy with R-DA-EPOCH, rituximab with dose adjusted EPOCH (etoposide, prednisone, vincristine, cyclophosphamide and doxorubicin) for 6 cycles. Postsurgery the platelet counts improved and the patient has completed first round of chemotherapy and is on follow up.

\section{DISCUSSION}

Malignant lymphoid tumors of female genital tract are rare, although ovaries are one of the common sites to be involved (25\%). ${ }^{4}$ There has been a debate as to whether lymphomas can arise de novo in the ovary; lymphoid 
aggregates do exist in normal ovarian tissues, which could give rise to such lesions.

Ovarian Non-Hodgkin's Lymphoma (NHL) accounts for $1.5 \%$ among all ovarian neoplasm and $0.5-1 \%$ among all NHL. Primary lymphoma of ovary is very rare, but secondary spread to ovary can occur in late stage of NHL. ${ }^{5}$

Lymphoma is a rare tumor of the ovary and its presence most commonly represents involvement in overt systemic disease, almost always of the non - Hodgkin's type.

The ovarian involvement in malignant lymphoma may be primary or secondary. ${ }^{6}$

Primary extra nodal lymphoma is less aggressive as there is an $80 \% 5$ years survival rate, whereas in secondary lymphoma it is $33 \% .^{7}$ So, it is of considerable importance to differentiate between them.

Fox et al, proposed the following criteria for the diagnosis of PONHL (primary ovarian non hodgkins lymphoma). ${ }^{8}$ (1) At the time of diagnosis, the lymphoma is clinically confined to the ovary and a complete investigation fails to reveal evidence of lymphoma elsewhere. However, an ovarian lymphoma can still be considered as primary if it has spread to immediately adjacent lymph nodes or if it has directly spread to infiltrate immediately adjacent structures. (2) The peripheral blood and bone marrow should not contain any abnormal cells. (3) If further lymphomatous lesions occur at sites remote from the ovary, then at least several months should have elapsed between the appearance of the ovarian and extra ovarian lesions.

Ovarian lymphoma is a rare entity. It may occur de novo or secondary to systemic disease and macroscopically appear as solid ovarian tumors.

Lymphoma of ovary is more common in women in $40 \mathrm{~s}$ but can occur in any age group..$^{9,10}$ The most common presenting symptoms/sign of patients with ovarian lymphoma are abdominal or pelvic mass or pain, weight loss, weakness, dyspnoea, vaginal bleeding, ascites and sometimes constitutional symptoms. However, fever of unknown origin for prolong duration should also be investigated on lines of malignancy if infectious causes are ruled out.

In this case the patient presented at 62 years of age with a history of fever for 3 weeks, and generalized symptoms of body ache, loss of appetite and weight loss of $5 \mathrm{kgs}$ in a month. There were no other significant features, and was investigated on lines of pyrexia of unknown origin. After ruling out all the possible infectious etiologies, PET-CT was done which showed metabolically active lesion, suspecting it to be uterine malignancy with no other significant finding pointing to any specific etiology. The patient was operated and intraoperatively was found to have solid ovarian tumor which was found to be malignant on frozen section.

The histological appearances of lymphoma in the ovary are generally similar to those seen in extra ovarian sites. The most common types of lymphomas encountered in the ovary are diffuse large-cell, burkitt and follicular lymphoma. However, these need to be distinguished from metastatic poorly differentiated carcinoma, adult granulosa cell tumor and dysgerminoma. Immunohistochemical studies help to differentiate between these tumors. ${ }^{7}$

B-cell and T-cell NHL may develop in ovary but B-cell is more common and stain-positive for pan B-cell marker CD20 whereas remains negative for $\mathrm{T}$-lineage marker CD3.

In this case immunohistochemical studies were performed for antigens like LCA, CD20, Bcl2, Bcl 6, CD3, Cytokeratin, etc. Diagnosed as high-grade B cell NHL- Germinal cell type with double expression type on histopathology and IHC.

The presence of positive staining for LCA in the histological specimen distinguishes malignant lymphoma from non-lymphoid neoplasm. ${ }^{11}$ Diffuse large B-cell lymphoma is most common sub-type of NHL and is the most common sub-type of Primary ovarian NHL. ${ }^{9}$ Patient with disease localized to one ovary usually do well with unilateral surgical resection followed by systemic chemotherapy. ${ }^{5}$ R-DA-EPOCH is the first line treatment for highly aggressive DLBCL. It is also important to know the type of lymphoma to initiate proper chemotherapy and to assess the prognosis of the case.

In the presence of an ovarian tumor, the possibility of ovarian NHL must be considered and its clinical, biological and/or radiological signs must be actively sought. ${ }^{12}$ For staging of primary lymphoma of ovary CT of chest, abdomen and pelvis is necessary. Bone-marrow biopsy is mandatory. ${ }^{11}$ Positron emission tomography (PET) with 18F-FDG (flouro deoxy glucose) is also done for staging and therapeutic assessment. ${ }^{13}$

\section{CONCLUSION}

Cancers can cause fever, but only fever with no other major symptom leading to a diagnosis of cancer is rare. This is an atypical presentation of an ovarian lymphoma. The prognosis of ovarian lymphomas is often poorer than nodal lymphomas because of delayed or inaccurate diagnosis. The best treatment is chemotherapy. Surgeons should be aware of this presentation also to have an early diagnosis and avoid radical surgeries and initiate proper treatment.

However, cancer of any type should be considered in a patient with prolonged fever and no evidence of infectious illness. The atypical presentations of ovarian 
lymphomas presenting only as fever with no other symptoms can lead to diagnostic dilemmas.

\section{ACKNOWLEDGEMENTS}

Authors would like to acknowledge the help extended by the Medical Director, department of obstetrics and gynecology, department of infectious diseases and department of pathology, Deenanath Mangeshkar Hospital and Research Centre, Pune, Maharashtra, India

Funding: No funding sources Conflict of interest: None declared

Ethical approval: Not required

\section{REFERENCES}

1. Hayakawa K, Ramasamy B, Chandrasekar PH. Fever of unknown origin: an evidence-based review. Am J Med Sci. 2012;344(4):307-16.

2. Cunha BA. Fever of unknown origin: clinical overview of classic and current concepts. Infect Dis Clin North Am. 2007;21(4):867-915.

3. Ekanayake CD, Punchihewa R, Wijesinghe PS. An atypical presentation of an ovarian lymphoma: a case report. J Med Case Reports. 2018;12(1):338.

4. Osborne BM, Robboy SJ. Lymphoma or Leukaemia Presenting as ovarian Tumours: An analysis of 42 cases. Cancer. 1983;52:1933-43.

5. Elharroudi T, Ismaili N, Errihani H, Jalil A. Primary lymphoma of the ovary. J Cancer Res Ther. 2008;4:195-6.

6. Chorlton I, Norris HJ, King FM. Malignant reticuloendothelial disease involving the ovary as a primary manifestation. A series of 19 lymphomas and 1 granulocytic sarcoma. Cancer. 1974;34(2):397407.

7. Crasta JA, Vallikad E. Ovarian lymphoma. Indian J Med Pediatric Oncol. 2009;30:28-30.

8. Fox H, Langley FA, Govan ADT, Hill SA, Bennett MH. Malignant lymphoma presenting as an ovarian tumour: a clinicopathological analysis of 34 cases. $\mathrm{Br}$ J Obstet Gynaecol. 1988;95(4):386-90.

9. Vang R, Medeiros LJ, Warnke RA, Higgins JP, Deavers MT. Ovarian non-Hodgkin's lymphoma: A clinicopathologic study of eight primary cases. Mod Pathol. 2001;14:1093-99.

10. Senol T, Doger E, Kahramanoglu I. Five cases of Non-Hodgkin B-cell Lymphoma of the Ovary. Case Rep Obst Gyn. 2014;1:Article ID 392758.

11. Vang R, Medeiros LJ, Fuller GN, Sarris AH, Deavers M. Non-Hodgkin's lymphoma involving the gynecologic tract. A review of 88 cases. Adv Anat Pathol. 2001;8:200-17.

12. Kaur N, Kaushi KR, Gulati A, Kaushal V, Chahal JS. Non-Hodgkin's Lymphoma presenting as an ovarian mass. Clin Cancer Invest J. 2014;3:231-4.

13. Komoto D, Nishiyama Y, Yamamoto Y, Monden T, Sasakawa Y, Toyama Y, et al. A case of nonHodgkin's lymphoma of the ovary: Usefulness of 18F-FDG PET for staging and assessment of the therapeutic response. Ann Nucl Med. 2006;20:15760 .

Cite this article as: Gokhale AN, Agarwal A, Purandare BD. Pyrexia of unknown origin: a rare presentation of primary ovarian lymphoma. Int $\mathrm{J}$ Reprod Contracept Obstet Gynecol 2020;9:2191-4. 